\title{
Micromechanical approach to wood fracture by three-dimensional mixed lattice-continuum model at fiber level
}

\author{
Marjan Sedighi-Gilani · Parviz Navi
}

Received: 17 November 2006/Published online: 13 April 2007

(C) Springer-Verlag 2007

\begin{abstract}
To investigate the fracture behavior of wood, the porosity and heterogeneities of its microstructure should be taken into account. Considering these features of wood microstructure in a continuum-based model is still a difficult problem and the lattice model might be an alternative. In the developed mixed lattice-continuum model, the probable crack propagation volume was modeled by defining a three-dimensional lattice of different beam elements and the other regions were considered as continuum medium. Different beam elements of lattice represented the earlywood fibers, latewood fibers, ray cells and bonding medium between the fibers. The proposed model was used to investigate the mechanism of mode I fracture in a small notched wood specimen in RL orientation. The resulting pre-peak and softening curve and also the crack opening trajectory in both cross-section and longitudinal-section in model were in good agreement with the experimental observations. This model shows the importance of considering the three-dimensional and distributed propagation of microcracks and main cracks in fracture stability. It was also shown that in mode I fracture, RL orientation, the main crack propagates in the earlywood ring.
\end{abstract}

\section{Introduction}

Different empirical strength theories, such as the maximum normal stress and strain theories, have been widely used in design of the structures. Strength of heterogeneous materials is controlled by their microstructure and local properties. Therefore, an increasing effort is being made in establishing more realistic strength criteria through micromechanical approaches.

M. Sedighi-Gilani · P. Navi ( $₫)$

Institute of Materials Science, Ecole Polytechnique Fédérale de Lausanne,

1015 Lausanne, Switzerland

e-mail: Parviz.Navi@epfl.ch 
Work in micromechanics usually starts with experiments and observation of physical phenomena of material behavior on a certain scale. The relevant scale depends on the phenomena in question. Next step requires mathematical modeling of the observed phenomena by idealizing the processes by simplification and by application of general principles of mechanics, laws of physics and chemistry. The goal is usually to understand the physics of the phenomena and the role of micromechanical effects on the phenomenological or macro level behavior.

Fracture behavior of wood is a function of morphology and mechanical properties of fibers, their bonding medium and heterogeneities at different scales. To understand the mechanism of crack propagation in the porous and heterogeneous microstructure of wood, different experimental and modeling approaches have been implemented. Experimental results indicate the predominant influence of the low stiffness of the bonding medium compared to the fibers (Schniewind and Centeno 1973). Scanning electron microscopy of fracture surfaces has shown that the bridging behind the crack tip is a toughening mechanism in wood fracture which contributes to its nonlinear behavior (Vasic et al. 2002). The alternation of earlywood and latewood fibers which have different microstructure, dimensions and stiffness can play an important role on the pattern of crack propagation (Boatright and Garrett 1983; Job and Navi 1996; Dill-Langer et al. 2002).

Despite the significant progress in computing power, modeling of the wood fracture still remains a difficult problem. There are some micromechanical approaches to consider the wood heterogeneities in the linear or non-linear continuum-based fracture models (Cramer and Goodman 1986; Holmberg et al. 1999). Due to the difficulties in implementing continuum-based fracture models to heterogeneous materials, simplified heterogeneities have been introduced in these models, which prevented the models from showing the major microscopic phenomena observed in the experiments.

Contrary to continuum-based fracture models, the morphological discrete, i.e. lattice model, might be a suitable approach to study the wood fracture. Lattice fracture model has been applied for investigating the mechanism of fracture in concrete and sandstone for several years (VanMier 1996; Schlangen and Garboczi 1997; Prado and VanMier 2003). Using this approach, Landis et al. (2002) developed a two-dimensional lattice model to study the mode I fracture of a notched wood specimen (Landis et al. 2002; Davids et al. 2003). The results of their model, which simulated the wood fracture at growth ring level (each wood bundle was represented by a lattice element), showed the convenience of morphological based models for investigating wood fracture and predicting the force-displacement curves and crack propagation paths.

As in the experiments shown, fracture in wood is a three-dimensional problem and is highly influenced by the heterogeneities at micro level. Consequently, developing a 3D model at fiber level is considered an essential step to understand the mechanism of wood fracture. In this study, the main objective is to investigate the role of different parameters that influence the wood fracture behavior. For this purpose, mode I fracture of a small softwood specimen in RL orientation is investigated by using a 3D lattice model at cellular level. Working with a 3D geometry allows us to monitor the crack propagation paths in all directions. To be 
able to study the fracture behavior at cellular level, the defined arrangement and size of the lattice elements should be similar to the earlywood and latewood fibers and their bonding medium. To minimize the computational costs and number of needed elements to define a 3D lattice geometry, a mixing technique is used; the critical volume where crack propagation is more probable (in front of the notch) is modeled by discrete lattice while the other regions are modeled by continuum elements.

\section{Materials and methods}

To investigate the mechanism of crack propagation and fracture in wood by using the lattice model, the material was discretized to a 3D lattice of beam elements with a mesh as fine as wood cellular structure. To reduce the number of needed elements to define the model geometry, a mixed technique based on coupling the lattice and continuum medium is used. For this purpose, crack path should be predicted at each step of analysis (as crack advances) and the region close to the crack trajectory should be modeled by lattice while the other parts are modeled by continuum elements.

Application of this approach to wood fracture in RL orientation is easier as the crack propagation paths are approximately predictable. The special microstructural formation of wood, parallel oriented fibers that are connected to each other by a bonding medium with considerably lower stiffness makes natural weak planes where crack has the tendency to propagate between them. In mode I fracture of a notched wood specimen in RL orientation, the crack propagates parallel to the fibers and in a limited volume in front of the notch tip (Mindess and Bentur 1986). Consequently in the mixed model, only this volume is replaced by lattice and the remaining parts are modeled by continuum elements (and a coarser mesh). In Fig. 1, the geometry and dimensions of the specimen (for simulating a direct tension fracture test) and the developed mixed lattice-continuum geometry are shown.

\section{Lattice geometry}

The defined lattice in model should represent the wood structure. Consequently, lattice should have different element sets that perform the role of the main microstructural elements of the wood material. The geometry and arrangement of these element sets should be similar to the wood microstructure at the considered level. In this model, it is assumed that the most critical elements of the wood microstructure in parallel to the fibers fracture (RL and TL orientation) are the parallel earlywood and latewood fibers, ray cells and bonding medium between the fibers, which has a lower stiffness.

The wood fibers are represented by series of parallel beam elements while the center of each beam is placed at the center of the fiber lumens. The length of these elements was considered to be $250 \mu \mathrm{m}$. Also the cross-sectional areas of different beams which represent the earlywood and latewood fibers were defined based on the wood microstructure in Fig. 2; $20 \times 40 \mu \mathrm{m}$ (in $\mathrm{R}$ and T directions) box beams with a thickness of $6 \mu \mathrm{m}$ in latewood fibers and $40 \times 40 \mu \mathrm{m}$ box beams with a thickness of 

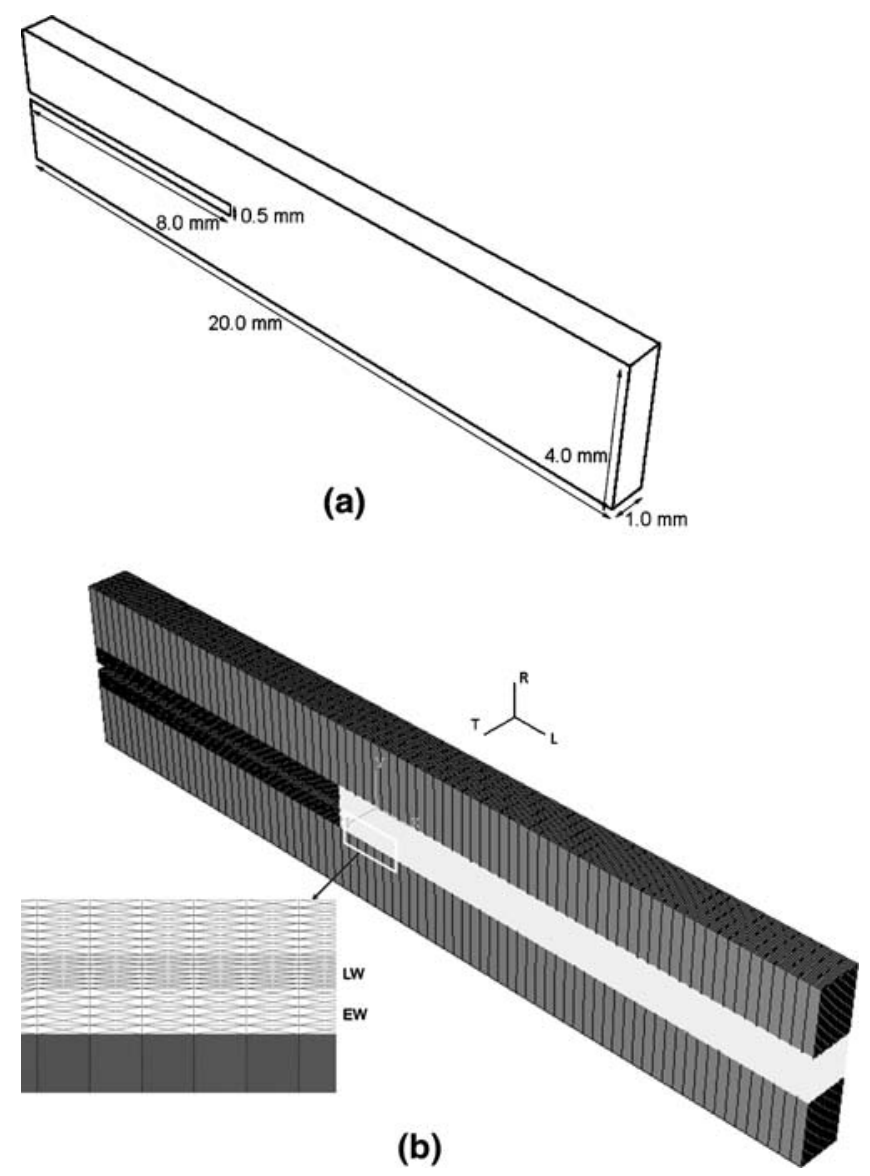

Fig. 1 a Geometry and dimensions of the specimen and $\mathbf{b}$ mixed lattice-continuum geometry; the volume in front of the notch is modeled by lattice elements

$2 \mu \mathrm{m}$ in earlywood fibers. Distances between the longitudinal axes of the beams are dependent on the cross-sectional dimensions of the fibers. In the $\mathrm{R}$ direction, distance between the longitudinal axes of earlywood and latewood beams was assumed to be 40 and $20 \mu \mathrm{m}$, respectively. Also in the $\mathrm{T}$ direction, this distance is assumed to be $40 \mu \mathrm{m}$.

The bonding medium between the wood fibers was represented by two sets of $S$ diagonal and L-diagonal beam elements and the ray cells were represented by direct beam elements in T direction (see Fig. 3). S-diagonal and L-diagonal elements were $50 \times 50 \mu \mathrm{m}$ rectangular beams and direct elements were $50 \times 50 \mu \mathrm{m}$ box beams with a thickness of $2 \mu \mathrm{m}$.

As each wood fiber in this model is represented by one beam element, investigating the intracellular propagation of crack is not possible. For the numerical calculation, B32 quadratic beam element of ABAQUS finite element code, defined 


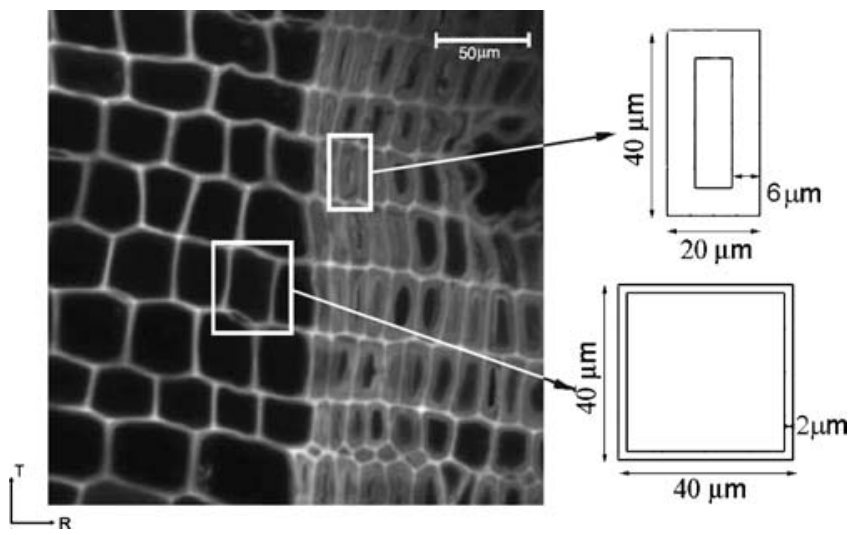

Fig. 2 Cross-sectional dimensions of earlywood and latewood beams used in the model are based on the wood microstructure

based on the Timoshenko beam theory is used. Based on the properties, dimensions and alignment of different beam element sets of lattice, its effective mechanical properties are determined.

\section{Mechanical properties}

The effective mechanical properties of the defined lattice and wood should be identical. Similar mechanical properties allow comparing the results of the fracture tests and of those obtained from the model. Moreover, to be able to use the mixed lattice-continuum model, consistency between the effective properties of the lattice and the assigned mechanical properties to the continuum is necessary.

In this study, the calculated stress-strain curves in the model are validated by comparing with the experimentally obtained stress-displacement curve by Vasic; mode I fracture test of notched spruce samples in RL orientation (Vasic 2000). In mode I fracture and parallel to the fibers directions, mechanical properties in the radial and tangential directions $\left(E_{\mathrm{R}}\right.$ and $\left.E_{\mathrm{T}}\right)$ are the most important properties in the fracture behavior. In the tested spruce specimen in Vasic's experiment, the $E_{\mathrm{R}}$ calculated from secant modulus of stress-displacement curve is about $280 \mathrm{MPa}$. To

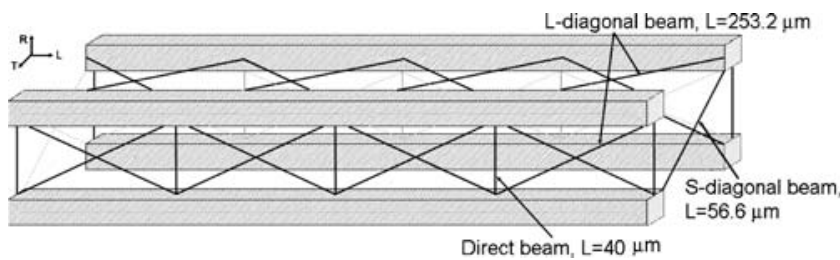

Fig. 3 Schematic representation of the main structural members of the defined 3D lattice, L-diagonal and $\mathrm{S}$-diagonal beams represent the bonding medium between the fibers and direct beams represent the ray cells 
get the mechanical properties in other directions, which cannot be calculated through the stress-displacement curve of this fracture test, the reported measured values in literature can be used (Hearmon 1948; Bodig and Jayne 1982). In Table 1, a summary of the reported properties of spruce in literature is given (Persson 2000).

The minimum reported value for $E_{\mathrm{R}}$ in Table $1(700 \mathrm{MPa})$ is more than the calculated $E_{\mathrm{R}}(280 \mathrm{MPa})$. Consequently, to be able to compare the simulation and experimental results, properties of lattice in the radial and tangential directions should be scaled to $40 \%$ of the minimum $E_{\mathrm{R}}$ and $E_{\mathrm{T}}$ values of Table 1, 280 and $160 \mathrm{Mpa}$, respectively. It should be mentioned that the main objective of this model is to investigate the mechanism of fracture. Consequently, this impreciseness of the input data should not be critical and could be enhanced after performing more experimental studies.

To adjust the effective mechanical properties of the lattice to the reference properties, the flexural stiffness of the lattice element sets are calculated by implementing an iterative approach. In each step of iteration, the lattice is solved for six uniform displacement loading cases (one for each column of the elasticity matrix in Voigt representation) by using ABAQUS. Characteristics of different beam element sets are changed to adjust the calculated elasticity matrix of the lattice against the reference properties of wood. In Tables 2 and 3, the appropriate characteristics of lattice element sets (calculated by iteration) and the effective properties of the defined lattice based on these inputs are presented.

\section{Failure criterion}

In lattice model, local fracture is simulated by analysis-time removal of the elements when their internal stress or strain exceeds a certain criterion. This predefined fracture criterion could be based on energy or strength. In this model, a tensile strength criterion was adopted which was based on the normal tensile strain in the beam elements; in each loading step, the lattice elements with higher normal strains than the predefined strain limit are removed.

Table 1 Range of variation of experimentally obtained mechanical properties of spruce, summarized by Persson (2000)

\begin{tabular}{lll}
\hline Coefficient & Maximum & Minimum \\
\hline$E_{\mathrm{L}}(\mathrm{MPa})$ & 25,000 & 6,000 \\
$E_{\mathrm{R}}(\mathrm{MPa})$ & 1,200 & 700 \\
$E_{\mathrm{T}}(\mathrm{MPa})$ & 900 & 400 \\
$G_{\mathrm{LR}}(\mathrm{MPa})$ & 700 & 600 \\
$G_{\mathrm{LT}}(\mathrm{MPa})$ & 600 & 500 \\
$G_{\mathrm{RT}}(\mathrm{MPa})$ & 70 & 20 \\
$v_{\mathrm{RL}}$ & 0.05 & 0.02 \\
$v_{\mathrm{TL}}$ & 0.025 & 0.01 \\
$v_{\mathrm{TR}}$ & 0.35 & 0.2 \\
\hline
\end{tabular}


The assigned strain limit of the lattice elements represents the strength of the wood fibers and the bonding medium. Strength of the bonding medium is the most influential parameter in fracture parallel to the wood fibers, but there is not enough information about its strength in literature. To overcome this problem, the strain limit of the elements which represent the bonding medium is estimated by comparing the obtained stress-strain curves from simulation (with different failure limits) with the experimental curves and choosing the failure limit which gives the best fit. Figure 4 shows the result of simulation for the strain limits presented in Table 4.

Defects and natural heterogeneities affect the strength and stiffness of the wood fibers and their bonding medium. This effect could be introduced to the model by randomly choosing the failure limit of each element from a normal distribution while its mean value represents the strength of the element set and the standard deviation represents the spread or variability in the strength value.

\section{Results and discussions}

\section{Force-displacement curve}

Each set of the elements in the defined lattice has a specific predefined function in providing the characteristics and properties of lattice. For example, shear properties of the lattice in the LR and LT planes are mainly defined by the flexural stiffnesses and the orientation of the L-diagonal elements in relation to the axial direction of the fibers ( $\mathrm{L}$ direction). Also, the lattice transversal property in the $\mathrm{R}$ direction is provided by the direct beam elements (ray cells) and the L-diagonal beams in the RL plane and in the $\mathrm{T}$ direction is provided by the S-diagonal beam elements and the L-diagonal beams in the LT plane. A parametric study on the strength of the different lattice elements could show the contribution of these elements in the fracture behavior of the whole model.

Comparison between the obtained stress-displacement curves from simulation with different strain limits of the S-diagonal and direct beams, while the strain limit of L-diagonal elements is unchanged, shows the important role of these elements in defining the peak stresses of simulation (Fig. 5a). In this example, the shown curves with [a] and [c] are the results of simulation when the strain limit of the S-diagonal

Table 2 Characteristic of different beam element sets of lattice (found by iteration)

\begin{tabular}{lccl}
\hline Element set & Area $(\mu \mathrm{m} 2)$ & Young's modulus $(\mathrm{MPa})$ & Poisson's ratio \\
\hline Earlywood beams & 304 & 2,350 & 0.3 \\
Latewood beams & 576 & 3,400 & 0.3 \\
Direct beams & 384 & 6,400 & 0.3 \\
S-diagonal beams & 2,500 & 450 & 0.3 \\
L-diagonal beams & 2,500 & 5,020 & 0.3 \\
\hline
\end{tabular}


Table 3 Effective properties of lattice based on the presented properties of elements in Table 2

\begin{tabular}{ll}
\hline Coefficient & Effective properties of lattice \\
\hline$E_{\mathrm{L}}(\mathrm{MPa})$ & 12,586 \\
$E_{\mathrm{R}}(\mathrm{MPa})$ & 280 \\
$E_{\mathrm{T}}(\mathrm{MPa})$ & 170 \\
$G_{\mathrm{LR}}(\mathrm{MPa})$ & 203 \\
$G_{\mathrm{LT}}(\mathrm{MPa})$ & 266 \\
$G_{\mathrm{RT}}(\mathrm{MPa})$ & 53 \\
$v_{\mathrm{RL}}$ & 0.015 \\
$v_{\mathrm{TL}}$ & 0.03 \\
$v_{\mathrm{TR}}$ & 0.17 \\
\hline
\end{tabular}

and direct beams are respectively $16 \%$ lower and higher than for the shown curve with $[b]$ and the failure limit of the other elements are as shown in Table 4. This figure shows that the peak stress of stress-displacement curve is mainly defined by the failure of S-diagonal and direct beams.

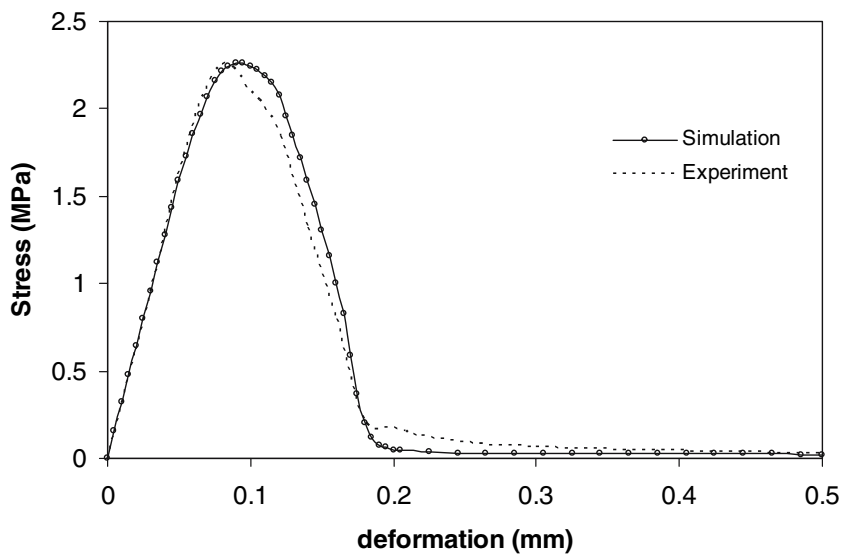

Fig. 4 Result of simulation with the presented failure limits in Table 4 gives the best fit to the obtained experimental result by Vasic (2000)

Table 4 Failure limit of different element sets of lattice

\begin{tabular}{llll}
\hline Element & Length $(\mu \mathrm{m})$ & Strain limit $(\%)$ & Standard deviation $(\%)$ \\
\hline S-diagonal and direct beams & 56.6 and 40 & 3 & 0.5 \\
L-diagonal beams & 253.2 & 0.67 & 0.11 \\
Earlywood and latewood fibers & 253 & 5 & 1 \\
\hline
\end{tabular}


The different form of the softening branches, while the assigned strengths to the L-diagonal beams are different, shows the important role of these elements in the post-peak behavior. In Fig. 5b, the shown curves with [a] and [c] are the results of simulation when the strain limit of the L-diagonal beams is respectively $50 \%$ lower and higher than for the shown curve with [b].

The role of the earlywood and latewood fiber beams in the fracture mechanism was investigated by comparing the results of simulation, while different strain limits were assigned to these elements (see Fig. 6a). These results indicate that the fiber elements have a negligible influence on the mechanism of mode I fracture in the directions parallel to the fibers.

Variability in the strength of the elements, which is introduced to the model by different standard deviation (SD) has an important effect on the simulation results. Figure $6 \mathrm{~b}$ shows that the peak stress of stress-displacement curve and the slope of softening branch for different considered standard deviations are different. Peak
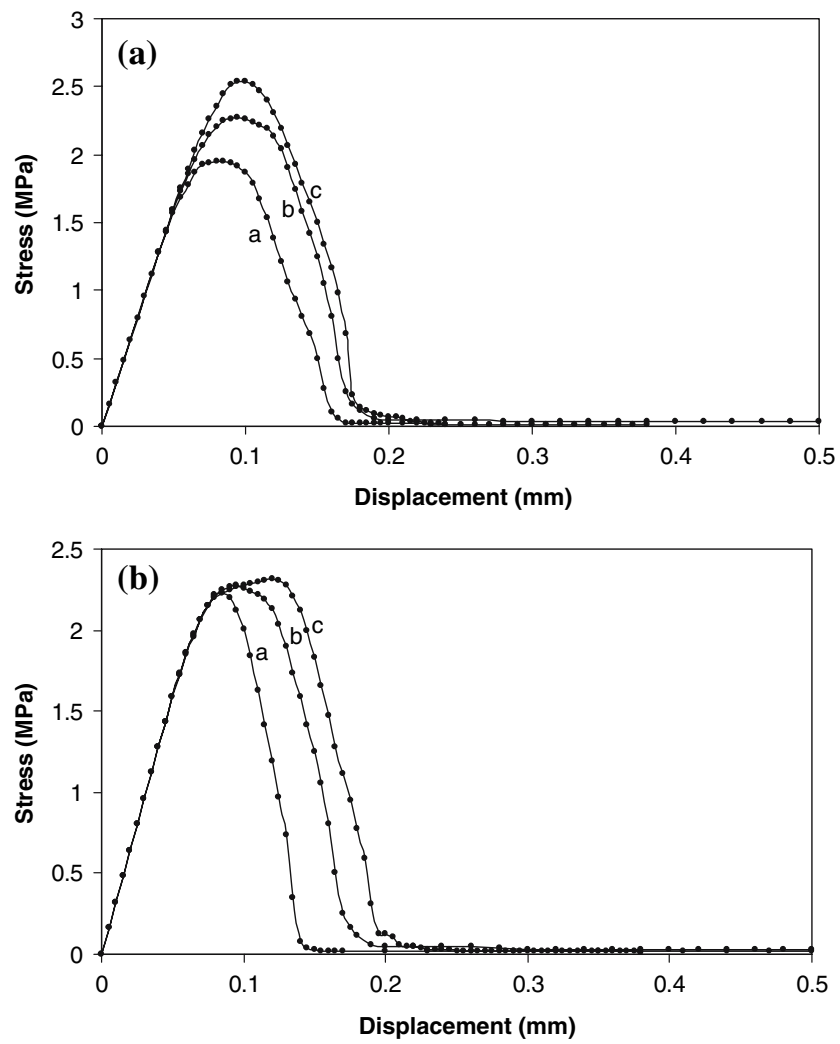

Fig. 5 Assigning different failure limits to the S-diagonal and direct elements changes the peak stress of the fracture curve and variation of failure limit in the L-diagonal elements changes the softening behavior: a the strain limit of S-diagonal and direct elements in curves $[a]$ and $[c]$ are $16 \%$ lower and higher than in curve $[b]$, b the strain limit of the L-diagonal beams in curves $[a]$ and $[c]$ is $50 \%$ lower and higher than in curve $[b]$ 
stress of the stress-displacement curve is reduced by considering larger standard deviations (interpreted as existence of more influential defects) and is increased by assigning smaller standard deviations.

One other important observation in Fig. $6 \mathrm{~b}$ is the successful simulation of a stable fracture test, even for non-variable failure limit $(\mathrm{SD}=0)$. This stability is attributed to the 3D heterogeneous geometry of the lattice, which is composed of different element sets with different flexural stiffnesses and settlement orders (horizontal, vertical or diagonal). In fact, the defined lattice allows the development of distributed microcracks, which are the origin of bridging and branching mechanism and fracture stability in model.

\section{$3 \mathrm{D}$ crack propagation}

In lattice model, removal of the critical elements from the lattice mesh during the simulation shows the location of developed cracks in the defined geometry. This is used in investigating the pattern of crack propagation in the cross-section and longitudinal-section of the specimen.
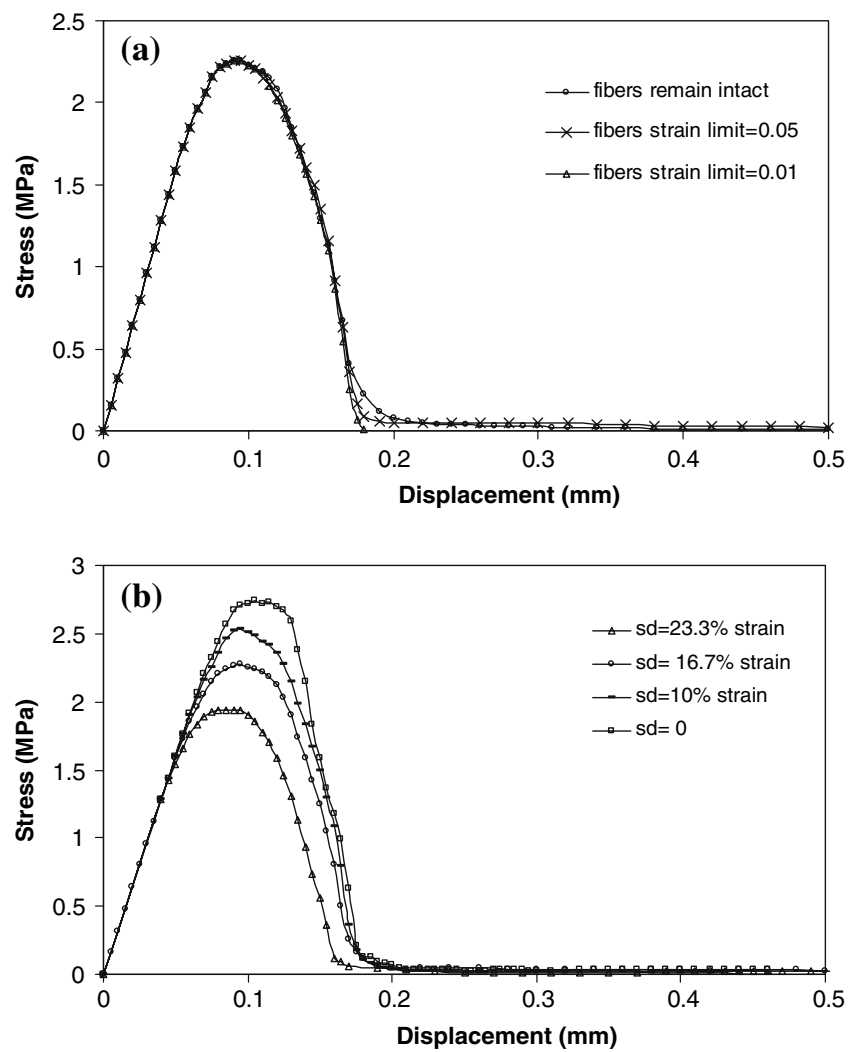

Fig. 6 a Similar shape of the stress-displacement curves shows the low importance of fiber elements in the results of mode I fracture test in RL orientation, $\mathbf{b}$ choosing the large standard deviations reduces the peak stress of the fracture curves and choosing the small one increases it 
Location of development of the microcracks and their influence on the material fracture were investigated. In Fig. 7, two different cross-sections of the lattice after application of $0.06 \mathrm{~mm}$ displacement (see stress-displacement curve in Fig. 4) are shown. Figure $7 \mathrm{~b}$ and $\mathrm{c}$ correspond to two cross-sections of lattice that are shown with $[\mathrm{A}]$ and $[\mathrm{B}]$ in Fig. 7a. For the same loading state, comparison between two cross-sections shows that the development of microcracks in the cross-section close to the notch tip initiates before the section far from it (section [A] is cracked while section [B] is still intact). This is attributed to the high stress concentration close to the notch tip for the defined geometry and loading condition.

Figure 8 shows propagated microcracks in the cross-section close to the notch tip (section [A] in Fig. 7a) under two other loading states. The developed microcracks in Fig. $8 \mathrm{a}$ and b correspond to $0.07 \mathrm{~mm}$ and $0.08 \mathrm{~mm}$ displacements applied in direct tension fracture test, which are indicated in Fig. 8c. This comparison shows that the development of microcracks initiates before the peak stress of the stressdisplacement curve and participates in forming the non-linear behavior of the material in this state. Also the first microcracks develop in the central earlywood region.

To investigate the mechanism of propagation of the main crack in different sections of lattice, three cross-sections close to the crack tip, under a known loading state were studied. Figure 9 shows these cracked cross-sections and their locations in the fractured profile, corresponding to $0.11 \mathrm{~mm}$ applied displacement. This figure indicates that in the mentioned fracture state, the main crack has propagated in the central earlywood region.
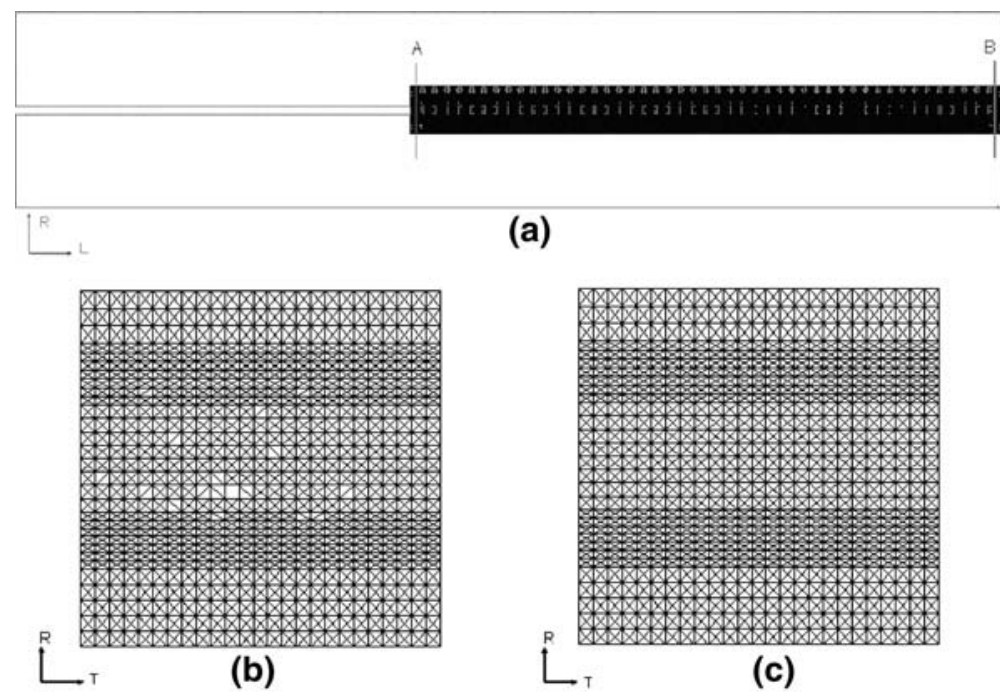

(a)

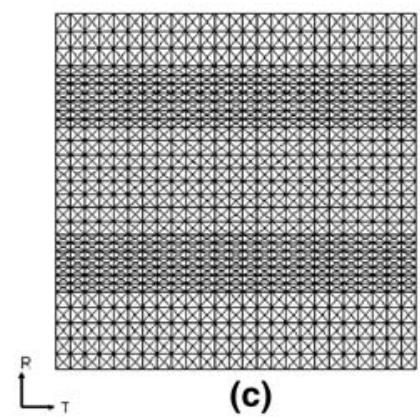

Fig. 7 Comparison between different cross-sections shows that first microcracks develop in the close region to the notch tip; b, c are the shown cross-sections with $[A]$ and $[B]$ in (a) 

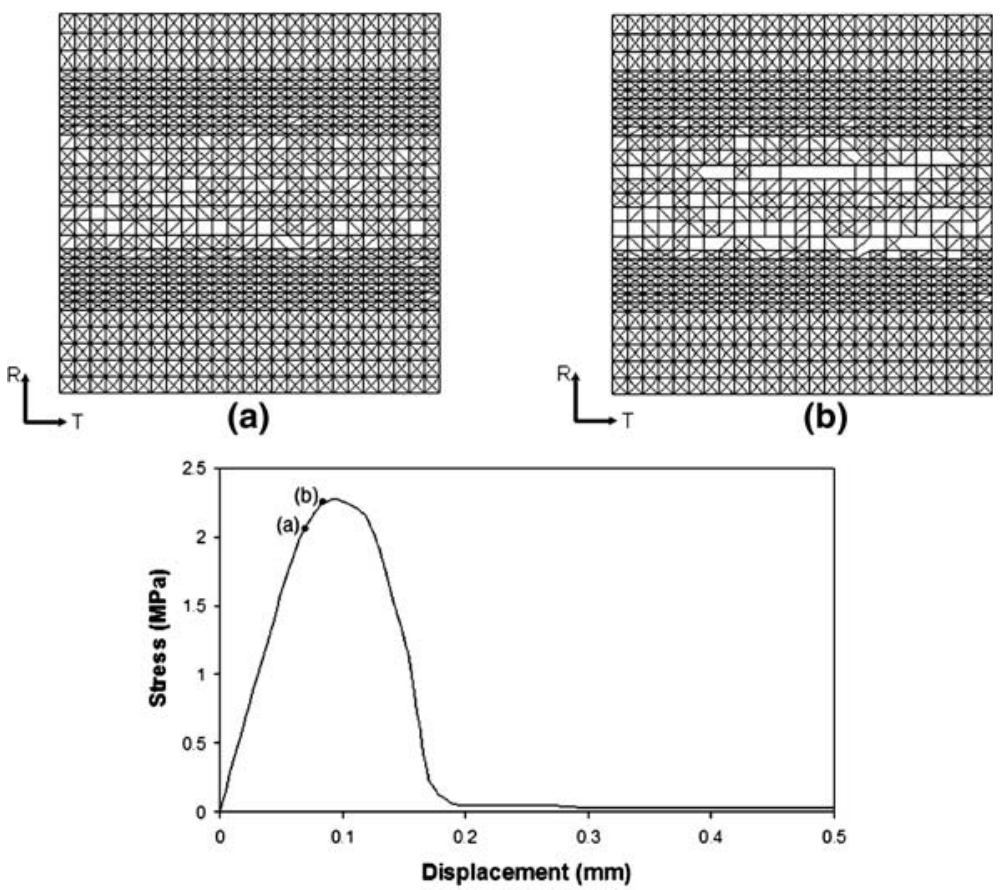

(c)

Fig. 8 Developed microcracks before peak stress in the notch tip (section $[A]$ in Fig. 7a); a, b developed microcracks under different loading states shown with the same notations in (c) corresponding to 0.07 and $0.08 \mathrm{~mm}$ displacement, c stress-displacement response

Initiation and propagation of crack in earlywood ring for the mode I fracture, RL orientation is confirmed by the former results of observation of the fractured spruce specimens, in compact-tension fracture test, by confocal laser scanning microscopy (Job and Navi 1996, Sedighi-Gilani et al. 2006). In order to investigate the network of microcracks and crack propagation path, the fracture test is stopped before complete fracture, cracked specimen is immobilized with a special technique (Job and Navi 1996) and a confocal laser scanning microscopy is used for observation of the fractured surfaces. This study showed that in mode I fracture, RL orientation, the main crack develops in earlywood region. Figure 10 indicates that cracks propagate within few cells width, in an earlywood ring and consist in mainly intercellular separation of the cells (the intracellular break of cell walls has been rarely observed). It means that the model incapability to consider the intracellular development of cracks does not have a critical influence on the results.

The microscopic observations showed that the initial damage is mostly localized around a few cells (Fig. 10) and expands until a continuous crack is formed. Because of the wide dispersion of microcracks that form in front of the crack tip, the classically defined fracture process zone could not be identified. However, it seems that the developed microcracks in these experiments are less dispersed than the early developed microcracks in the model geometry (see Fig. 8). This could be 


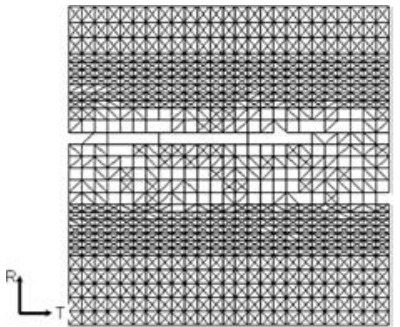

(a)

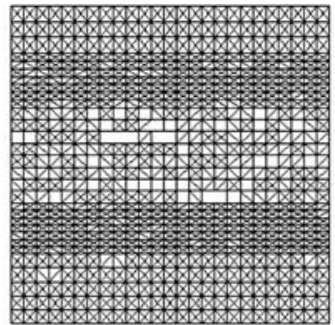

(b)

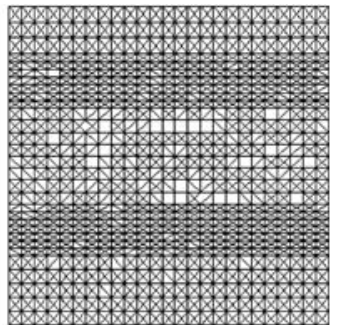

(c)

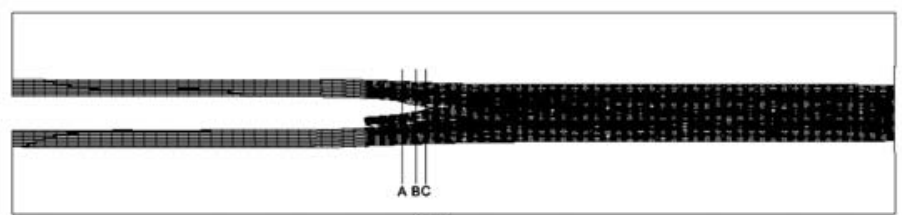

(d)

Fig. 9 Crack pattern in the cross-sections close to the crack tip under a given loading state after the peak stress; a, b, c Different cross-sections at locations $[A],[B]$ and $[C]$ in the central longitudinal-section $(\mathbf{d})$

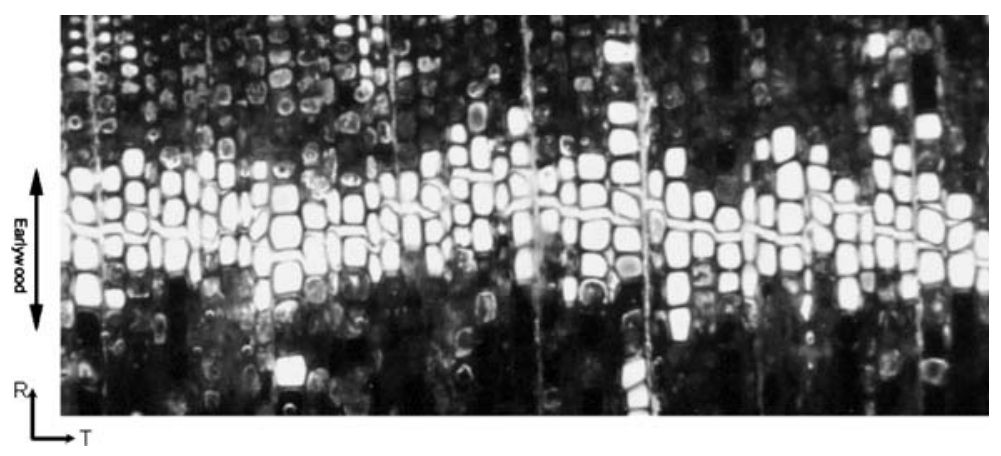

Fig. 10 Mode I fracture in RL orientation consists of intercellular separation of the earlywood fibers while intracellular fracture of cell walls is rarely observed

attributed to the different distribution of internal stresses in the two different fracture tests, direct tension fracture test in the model and compact-tension fracture test in the experiments. The abrupt heterogeneities of the model geometry (compared to the wood nature) and the need to work with a more detailed mesh could be the other reason for this inconsistency.

Figure 11 shows the process of initiation and propagation of crack in the central longitudinal-section of the defined geometry, for different fracture states. Figures $11 \mathrm{a}-\mathrm{e}$ show that a main crack propagated forward until the secondary cracks appeared (in Fig. 11f). After this state, branching and bridging become the more important mechanisms of stress transfer from one part of the material to the other (see Fig. $11 \mathrm{~g}, \mathrm{~h}$ ), until the specimen shows a very small resistance to the 


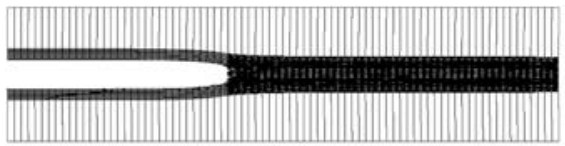

(a)

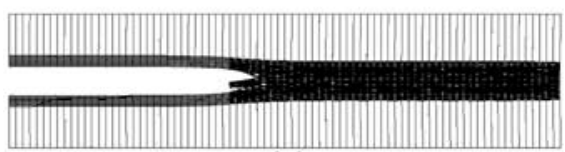

(c)

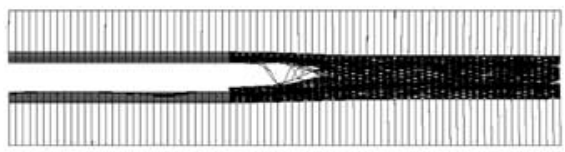

(e)

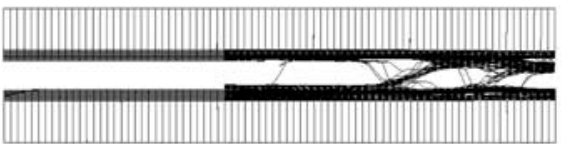

(g)

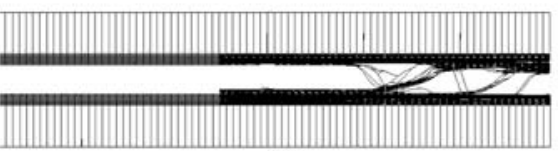

(h)

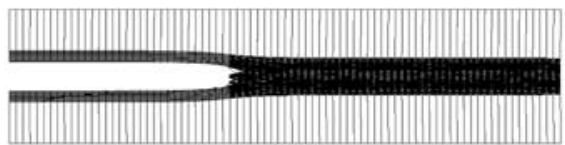

(b)

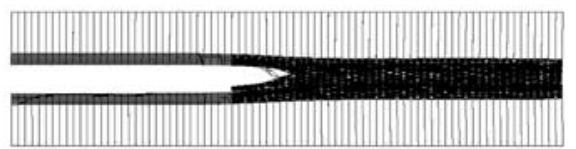

(d)

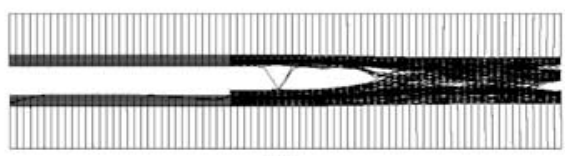

(f)

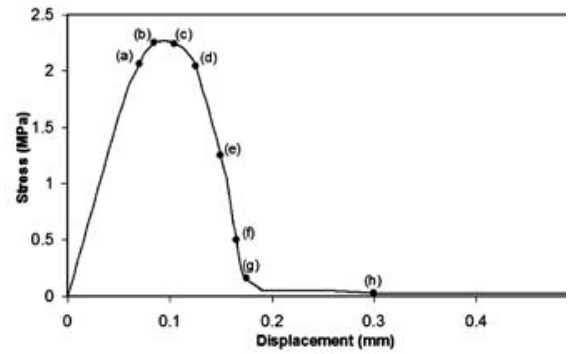

(i)

Fig. 11 a-h Crack advance process in the central longitudinal-section of the specimen under the shown loading states in (i)

applied displacements. However, these mechanisms were not clearly visible in these figures, as only the central longitudinal section of the specimen was shown and the elements out of the cut section were hidden.

Figure 12 shows the whole 3D fractured geometry of the specimen under the same loading state as in Fig. 11h. The elements which still work around the main crack show that the branching and bridging mechanisms provide the long tail of the stress-strain curve in softening state.

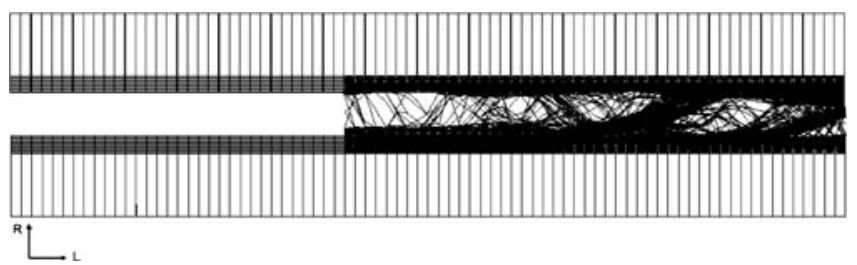

Fig. 12 Branching and bridging mechanism around the localized crack in whole fracture geometry 


\section{Conclusions}

A mixed lattice-continuum model was developed to investigate the mechanism of crack propagations in wood under mode I and RL orientation, while its heterogeneities and porosity were taken into account. The obtained stressdisplacement response and crack opening trajectory in model were in good agreement with the experimental evidences.

The presented model can simulate the pre-peak non-linearity of the stressdisplacement curve and the post-peak strain softening (fracture stability). Agreement between the slopes of the strain softening branches in numerical simulation and experimental results is attributed to the capability of this model to consider the microstructural heterogeneities in wood and the 3D mechanism of crack propagation in this microstructure.

The role of different element sets of the defined lattice geometry on the mechanism of fracture was investigated by assigning different failure limits to these elements and comparing the resulting stress-displacement curves. These investigations indicate that, contrary to the bonding medium, the fiber elements have negligible influence on the mechanism of mode I fracture parallel to the direction of the fibers. The direct and S-diagonal elements define the peak stress of the stressdisplacement curve and L-diagonal elements play an important part in post-peak behaviour.

The model showed the location of the early developed microcracks as well as the crack pattern in both longitudinal-section and cross-section in different fracture states. Detecting the crack propagation paths in the lattice cross-sections showed that in mode I fracture, RL orientation, cracks propagate in the earlywood ring. This is confirmed by comparison with the results of microscopic (CLSM) observations of a fractured spruce specimen, in mode I and RL orientation.

All these points show that this micromechanical approach and the developed 3D mixed lattice-continuum model are appropriate tools to investigate the mechanism of fracture in the porous and heterogeneous materials like wood. However, the results of the lattice model are dependent on the order, dimensions and alignments of different elements of the defined geometry, the mechanical properties of each element and the failure criteria. Consequently, characterization of these parameters is the most essential part of the lattice model and requires to be enhanced further.

Acknowledgment The financial support of the Swiss Federal Office of Education and Science in the framework of COST E35 is acknowledged with gratitude.

\section{References}

Boatright SWJ, Garrett GG (1983) The effect of microstructure and stress state on the fracture behavior of wood. J Materials Sci 18:2181-2199

Bodig J, Jayne B (1982) Mechanics of wood and wood composites. Van No Reinhold Company, New York

Cramer SM, Goodman JR (1986) Failure modelling: a basis for strength prediction of lumber. Wood Fiber Sci 18:446-459 
Davids WG, Landis EN, Vasic S (2003) Lattice models for the prediction of loading-induced failure and damage in wood. Wood Fiber Sci 35:120-134

Dill-Langer G, Lütye S, Aicher S (2002) Microfracture in wood monitored by confocal laser scanning microscopy. Wood Sci Technol 36:487-499

Hearmon R (1948) The elasticity of wood and plywood. In: Special report on forest products research, HMSO London, no 7, p 87

Holmberg S, Persson K, Petersson H (1999) Non-linear mechanical behavior and analysis of wood and fiber material. Comput Struct 72:459-480

Job L, Navi P (1996) Microscopic analysis of crack propagation in softwood, model I and II. International COST 508 wood mechanics conference Stuttgart, Germany

Landis EN, Vasic S, Davids WG, Parrod P (2002) Coupled experiments and simulations of microstructural damage in wood. Exp Mech 42:1-6

Mindess S, Bentur A (1986) Crack propagation in notched wood specimens with different grain orientations. Wood Sci Technol 20:145-155

Persson K (2000) Micromechanical modeling of wood and wood fiber properties. Doctoral Thesis, LUND, Sweden

Prado EP, VanMier JGM (2003) Effect of particle structure on mode I fracture process in concrete. Eng Fracture Mech 70:1793-1807

Schlangen E, Garboczi EJ (1997) Fracture simulations of concrete using lattice models: computational aspects. Eng Fracture Mech 57:319-332

Schniewind AP, Centeno JC (1973) Fracture toughness and duration of load factor I. Six principal systems of crack propagation and the duration factor for cracks propagating parallel to grain. Wood Fiber 5(2):152-159

Sedighi-Gilani M, Job L, Navi P (2006) Three-dimensional modeling of wood fracture in mode I, perpendicular to the grain direction, at fiber level. Wood Material Sci Eng 1:52-58

VanMier JG (1996) Fracture processes of concrete: assessment of material parameters for fracture models. CRC Press, West Palm Beach

Vasic S, Smith I, Landis E (2002) Fracture zone characterization-micromechanical study. Wood Fiber Sci 34:42-56

Vasic S (2000) Applications of fracture mechanics to wood, Doctoral, Fredericton, N.B. University of New Brunswick 